\title{
Influence of Xpand Nitric Oxide Reactor, L-Arginine Alpha-Ketoglutarate, and Caffeine Supplementation on Calf Muscle Re-Oxygenation During and after Acute Resistance Exercise
}

\author{
Brittany C. Collins ${ }^{1,2}$, Ryan Sapp ${ }^{1}$, John LaManca ${ }^{1}$, Christopher Wolff ${ }^{1,3}$, Edwin Miranda ${ }^{1}$, \\ Vincent Gutierrez ${ }^{1,4}$ and Scott A. Mazzetti ${ }^{1, *}$ \\ ${ }^{1}$ Salisbury University, Salisbury, MD, USA \\ ${ }^{2}$ University of Minnesota, Minneapolis, MN, USA \\ ${ }^{3}$ Ball State University, Muncie, IN, USA \\ ${ }^{4}$ National Institute on Aging, Baltimore, MD, USA
}

\begin{abstract}
Xpand Nitric Oxide Reactor is a 'cocktail' supplement proposed to improve skeletal muscle blood flow via arginine's effect on nitric oxide synthesis and vasodilation. Two other major ingredients, caffeine and creatine, cause vasoconstriction, which could potentially counteract the proposed hemodynamic effects of arginine. The purpose of this study was to examine the influence of Xpand Nitric Oxide Reactor on muscle re-oxygenation after resistance exercise compared to supplementation with constituent ingredients L-arginine alpha-ketoglutarate and caffeine. Nine recreationally active men $(21 \pm 1 \mathrm{y})$ performed 3 sets of 20 repetitions of seated single-leg calf raise at $60 \% 1-\mathrm{RM}$ with 3 min rests. The same calf raise exercise was performed following 4 separate supplementation conditions: L-arginine alpha-ketoglutarate (AAKG), caffeine (CAFF), Xpand Nitric Oxide Reactor (XPAND), and placebo (PLAC). Soleus muscle re-oxygenation time was measured before, during, and immediately after exercise using near infrared spectroscopy. Supplementation with XPAND (0.43 \pm 0.03$)$, AAKG $(0.34 \pm 0.02)$, and CAFF $(0.45 \pm 0.05)$ did not significantly affect muscle re-oxygenation halftime (minutes) compared to placebo (0.35 \pm 0.04$)$. An arginine containing 'cocktail' supplement did not affect skeletal muscle re-oxygenation after resistance exercise, possibly due to a wash-out effect caused by the multiple ingredients.
\end{abstract}

Keywords: Ergogenic, blood flow, oxygen delivery, supplements, vasomotor, athletic performance, near infrared spectroscopy.

\section{INTRODUCTION}

Nitric Oxide (NO) is an endogenously produced gas that helps to maintain arterial vasodilation and regulate blood flow at rest via its actions on vascular smooth muscle [1-3]. During exercise, NO production increases to enhance blood flow and ensure that sufficient oxygen is delivered to working skeletal muscle [4]. Enhanced blood flow during exercise could also increase nutrient delivery to (e.g. amino acids, glucose, creatine, etc.), and metabolic waste removal from skeletal muscle (e.g. $\mathrm{CO}_{2}$, nitrogen, bicarbonate, etc.). Because $\mathrm{NO}$ is synthesized from the semi-essential amino acid L-arginine [5], this potentially ergogenic mechanism has become an important selling point on which arginine-containing nutritional supplements are claimed to increase "muscle pumps" during resistance exercise, and muscle hypertrophy and performance gains with training. As a result, several products containing L-arginine or L-arginine alpha-ketoglutarate have become increasingly popular and continue to amass millions of dollars in sales annually.

*Address corresponding to this author at the Salisbury University, Laboratory for Human Performance, Maggs 216, 1101 Camden Ave., Salisbury, MD 21801, USA; Tel: 410677 0151; Fax: 4105436434 ;

E-mail: samazzetti@salisbury.edu
Xpand Nitric Oxide Reactor is a 'cocktail' supplement that is intended for athletes and others who wish to increase muscle hypertrophy and enhance resistance exercise performance, and the ergogenic potential of Xpand has been previously investigated [6]. Xpand contains L-arginine alpha-ketoglutarate (AAKG), as well as other ingredients including caffeine and creatine monohydrate. The AAKG is intended to stimulate NO production for vasodilation [5], while caffeine may increase blood pressure, heart rate, and endurance performance [7], and creatine has been shown to increase strength, power, and recovery during shortburst resistance exercise [7-8]. Because creatine and caffeine have been demonstrated to have beneficial effects on skeletal muscle performance, it is logical that supplement companies combine these ingredients with the potential vasodilator arginine in an attempt to exploit the combined activities of these compounds. Thus the strategy of supplement companies is to promote that when taken just prior to exercise, Xpand Nitric Oxide Reactor and other similar products should increase exercise vigor and blood flow, thereby increasing nutrient and creatine delivery to skeletal muscle. The resultant enhanced re-oxygenation and creatine delivery to muscle could also improve recovery 
between intense contractions, which might further increase exercise performance [7, 9-12].

Alone, L-arginine supplementation has resulted in improved gains in strength and anaerobic power compared to placebo following twelve weeks of resistance training in healthy men [13]. A possible explanation for these ergogenic effects could be related to L-arginine's role in NO synthesis, as NO-mediated vasodilation has been shown to partly account for the greater muscle blood flow during exercise after a period of training [1, 14]. To further test this observation, Little et al. (2008) compared the effects of ten days of supplementation with Xpand Nitric Oxide Reactor, creatine, or placebo on bench press endurance and Wingate power, in what is currently the only study that has examined the ergogenic potential of Xpand [6]. The results showed that only the Xpand Nitric Oxide Reactor group improved in Wingate peak power, prompting the authors to propose that arginine and creatine may be more beneficial when taken together. Despite the reported benefits of L-arginine as an ergogenic aid, it remains unclear whether supplementation with L-arginine or L-arginine containing products alters hemodynamic properties in skeletal muscle for improved re-oxygenation.

Contrary to the product claims, the main ingredients of 'cocktail' supplements like Xpand could have conflicting effects on arterial and capillary vasomotor control. AAKG is believed to increase NO production and vasodilation [15], yet two other ingredients creatine and caffeine may decrease vasodilation [8, 16]. Theoretically, the cumulative effect of these three ingredients could be decreased or unaltered vasodilation and blood flow. In fact, Bloomer et al. (2010) reported no difference in skeletal muscle oxygen saturation following pre-exercise supplementation with three different L-arginine containing 'cocktail' supplements compared to placebo [17]. To date, however, no study in humans has directly examined the effects of an L-arginine containing 'cocktail' supplement compared to its constituent ingredients on skeletal muscle re-oxygenation following intense resistance exercise. Thus the purpose of this study was to compare muscle re-oxygenation during and after acute resistance exercise among Xpand Nitric Oxide Reactor (XPAND), caffeine (CAFF), L-arginine alpha-ketoglutarate (AAKG), and placebo (PLAC) supplementated conditions. We hypothesized that the time to re-oxygenate skeletal muscle would decrease following AAKG, increase following CAFF, and would not change following XPAND supplementation when compared to PLAC.

\section{METHODS}

\section{Subject Participation}

Ten resistance trained men (21 $\pm 1 y)$ from the Salisbury University community volunteered to complete the requirements of this study, but one subject withdrew due to circumstances unrelated to the study leaving a final n-size equal to nine. Each participant had a minimum of two years resistance training experience. Participants were non-smoking, free from any cardiovascular or metabolic diseases, and cleared to complete the study and consume the supplements by the study physician. Medical history questionnaires were completed by the subjects and reviewed by the physician to determine possible contraindications for supplementation. Subjects were asked to refrain from nutritional supplements and resistance exercise during the entirety of the study, as well as stimulants (e.g., caffeine) and alcohol for 72 hours prior to each protocol. Subjects were asked to not ingest anything 3 hours prior to their trial. Subjects were excluded from the study if they were currently using supplements or had discontinued supplement use in the previous 3 months. The Salisbury University Committee on Human Research approved this study, and all participants provided verbal and written consent.

\section{Study Design}

This was a randomized, placebo controlled, double blind study. The subjects' height, weight, and body composition (7-site skinfold method) were measured during the first visit prior to 1-repetition maximum (1$\mathrm{RM}$ ) testing. Each subject's dominant leg 1-RM was determined on a seated calf-raise machine (Body Masters Sports Industries, Rayne, LA) following NSCA guidelines for 1-RM testing [18]. Following 1-RM testing, subjects were further familiarized with single leg calf raises with the near infrared spectrometer (RunMan, NIM Inc., Philadelphia, PA). Exactly one week after 1-RM testing, subjects began experimental protocols (see Exercise Protocols below).

\section{Supplementation}

Participants orally ingested one of four supplements in solution with similar coloration: L-arginine alphaketoglutarate powder $\left(0.075 \mathrm{~g} \cdot \mathrm{kg}^{-1} \cdot \mathrm{day}^{-1}\right)$ (Olympian Labs Inc., Phoenix, AZ), pulverized Jet Alert caffeine 


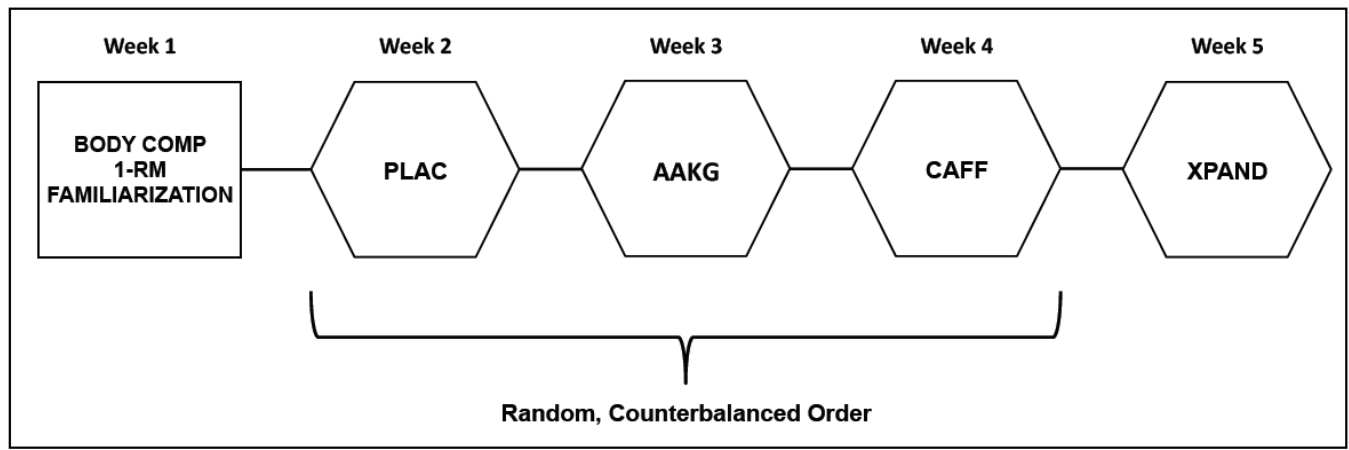

Figure 1: Study design timeline. Body Comp = body composition, 1-RM = 1-repetition maximum, PLAC = placebo, AAKG = $\mathrm{L}$-arginine alpha-ketoglutarate, CAFF $=$ caffeine, XPAND $=$ Xpand Nitric Oxide Reactor.

pill (100mg) (Bell Pharmaceuticals, Minneapolis, MN), Xpand Nitric Oxide Reactor powder $(0.35 \mathrm{~g} / \mathrm{kg})$ (Dymatize Nutrition, Farmers Branch, TX), and placebo $\left(1 \mathrm{~g} \cdot \mathrm{kg}^{-1} \cdot \mathrm{day}^{-1}\right.$ sugar free fruit punch powder) (Wal-Mart Stores Inc., Bentonville, AR). These amounts were selected because they reflect the specific ingredient amounts in one serving of XPAND, and have been used in previous research [6]. The PLAC was similar in caloric content to the XPAND, and the placebo fruit punch powder was added to CAFF and AAKG to match caloric content and color. AAKG, CAFF, and PLAC were taken in a randomized, counterbalanced order. Because creatine requires a wash-out period after use, XPAND was consumed during the last testing session for all subjects, and this was also the reason for not including a creatine only protocol. Subjects were instructed that the order of all trials would be randomized, and were not aware that XPAND would be last (Figure 1).

\section{Exercise Protocol}

All testing protocols were identical and performed at the same time of day (1600hr) exactly one week apart for each subject. Consistent with supplement instructions and previous research [6], testing consisted of consuming the assigned supplement 45 minutes prior to exercise (Figure 2). Subjects were then fitted with the near-infrared apparatus secured by an elastic bandage on the lateral lower limb over the soleus muscle and subsequently completed three sets of twenty repetitions of single leg calf raises with three minutes of rest between sets. All calf-raise repetitions were completed with $60 \%$ of 1 -RM as determined from 1-RM testing and familiarization. Subjects lowered the loads in $2 s$ and raised the loads in $2 s$ guided by the cadence of a metronome.

\section{Near Infrared Spectroscopy}

The near infrared spectrometer is a non-invasive device that monitors the relative de-oxygenation of hemoglobin and blood volume used to determine the oxygen delivery to the subject tissue. It emits a white light into skeletal muscle that is read on the nearinfrared spectrum. The spectrometer shows an optical density, which decreases when the muscle is deoxygenated. Near infrared spectrometry has been previously validated [19-23]. In addition, intra-subject muscle re-oxygenation coefficients of variation (CV) were calculated during the familiarization session to ensure its reliability $(\mathrm{CV}=3.7 \%)$.

\section{Statistical Analysis}

A repeated-measures two-way analysis of variance was used to compare amongst supplement groups with all sets combined. A separate ANOVA was used for

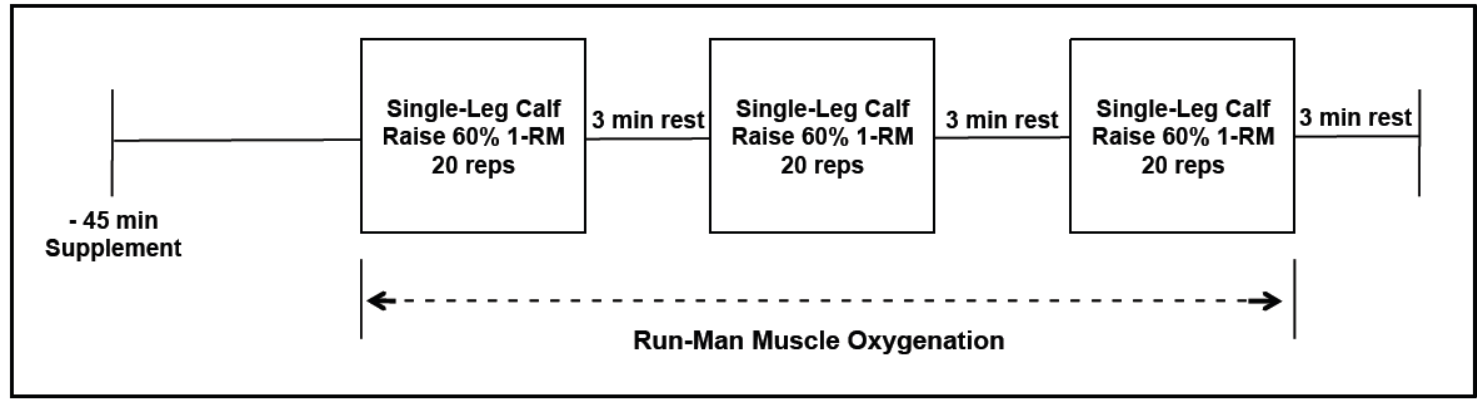

Figure 2: Single-leg calf raise exercise protocol. 
between sets and relative percent change. A paired student's t-test was used to compare pre- and postexercise optical density values. Data are reported as means \pm standard error (MEANS \pm SEM). Statistical power ranged from 0.80 to 0.97 at a $P$-value equal to 0.05 . Significance for this study was set at $P \leq 0.05$.

\section{RESULTS}

\section{Subject Characteristics and Reported Side Effects}

Subjects were weight stable throughout the course of the study. Moreover, there were no significant differences between subjects for each of these measures (Table 1). In addition, three subjects reported side effects such as itchy skin, nausea, tachycardia, and flushed face, which has also been previously reported [24]. Subjects reported that these symptoms distracted them mostly during the 45 minute pre-exercise period (since they were required to sit still), and that they were hardly noticeable during the exercise and did not adversely affect their exercise performances.

Table 1: Subject Characteristics Measured at Baseline (Mean \pm SEM)

\begin{tabular}{|c|c|}
\hline Baseline Measurements & $\mathbf{n}=\mathbf{9}$ Males \\
\hline \hline Age $(\mathrm{yrs})$ & $21.00 \pm 0.37$ \\
\hline Height $(\mathrm{cm})$ & $178.1 \pm 2.99$ \\
\hline Weight $(\mathrm{kg})$ & $91.05 \pm 3.87$ \\
\hline BMl & $27.44 \pm 1.86$ \\
\hline Body Fat $(\%)$ & $14.06 \pm 2.74$ \\
\hline $1-\mathrm{RM}(\mathrm{kg})$ & $76.22 \pm 3.75$ \\
\hline $60 \% 1-\mathrm{RM}(\mathrm{kg})$ & $42.78 \pm 3.32$ \\
\hline
\end{tabular}

Note: BMI, body mass index; 1-RM, one repetition maximum.

\section{De-Oxygenation of the Soleus Muscle During the Exercise Protocol}

We validated our experimental protocol to deoxygenate the soleus muscle and to return to baseline after rest (Figure 3). Figure $\mathbf{3}$ is representative data for each of the subject's optical densities measured by near infrared spectrometry throughout the experimental protocol. The exercise optical density was significantly decreased compared to pre- and post-exercise values $(P=0.000002)$.

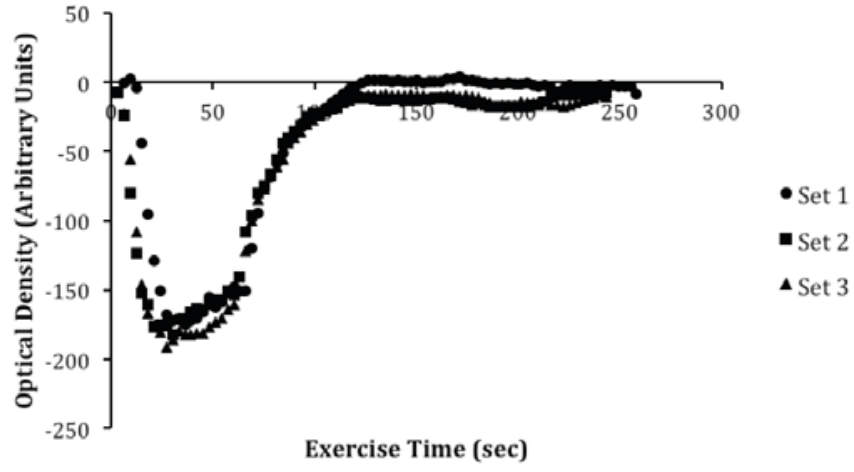

Figure 3: Blood oxygenation (optical density) during each set of seated-calf raise exercise.

\section{Re-Oxygenation Half-Time $\left(\mathrm{t}_{1 / 2}\right)$}

Skeletal muscle blood flow was significantly $(\mathrm{P}<$ $0.05)$ decreased during exercise compared to resting and recovery values. Despite a trend, there were no significant differences in subjects' half-times among the supplement groups $(P=0.07)$ (Figure 4). There were also no significant differences in half-times for each set among the supplement groups $(P=0.07)$ (Figure 5). Interestingly, AAKG supplementation consistently had the shortest half-time and CAFF supplementation consistently led to the longest half-time during all three sets and with all sets combined (Figures $\mathbf{4}$ and $\mathbf{5}$ ). This is also depicted in the percent changes of the halftimes when relative to placebo (Figure 6). Again, there were no significant differences in the percent changes among the supplement groups $(P=0.1)$. Overall, supplementation of CAFF, AAKG, and XPAND did not affect re-oxygenation of the soleus muscle after exercise.

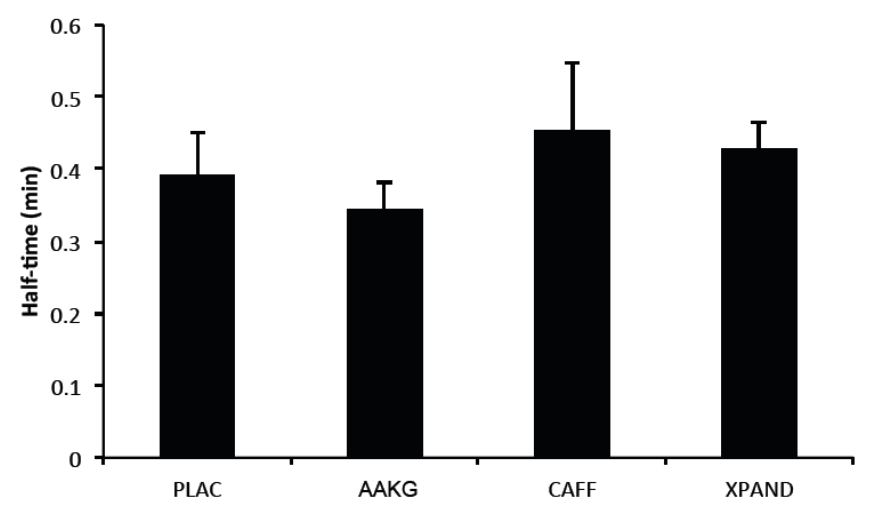

Figure 4: Blood re-oxygenation half-times ( $\mathrm{min}$ ) for all sets combined for each supplement.

\section{DISCUSSION}

To compare the effects of an L-arginine containing 'cocktail' supplement versus its constituent ingredients, we tested three different supplementation conditions 


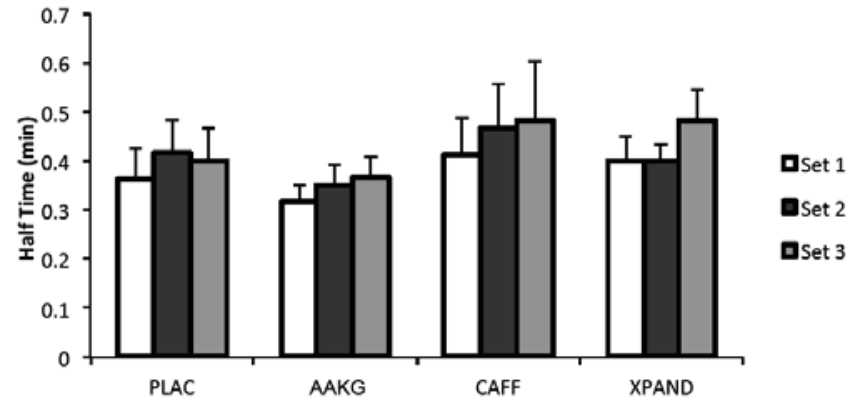

Figure 5: Blood re-oxygenation half times ( $\mathrm{min}$ ) for each set of seated calf-raise exercise for each supplement.

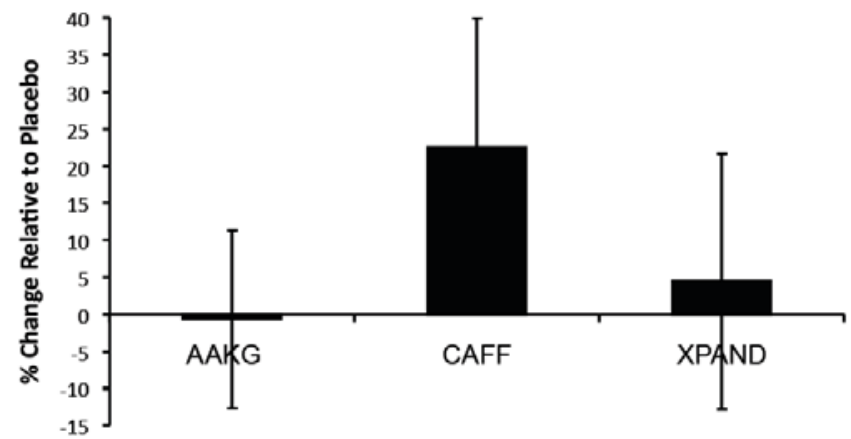

Figure 6: The percent change in blood re-oxygenation halftimes relative to the placebo supplement.

(AAKG, CAFF, and XPAND) on soleus muscle reoxygenation during and after resistance exercise in men. We predicted that AAKG would decrease and CAFF would increase re-oxygenation time, and that the combination of ingredients in XPAND would elicit no effect. As expected, XPAND supplementation had no effect on calf muscle re-oxygenation. However, contrary to our hypotheses, the individual responses of AAKG and CAFF were also not significant.

This was the first study to compare calf muscle reoxygenation time after resistance exercise in men who performed the same protocol on three different occasions with a 'cocktail' supplement compared to two constituent ingredients. This study design allowed us to examine the effects of arginine and caffeine individually, and as combined ingredients of Xpand Nitric Oxide Reactor versus a control condition. We found that Xpand Nitric Oxide Reactor did not change re-oxygenation half-time compared to placebo. Our study is unique, because most studies that have examined supplementation with L-arginine 'cocktail' supplements have assessed performance variables such as muscle power, time to fatigue, muscle fatigue recovery, anaerobic power, and muscle strength [25]. One study by Bloomer et al. (2010) examined the effect of 'cocktail' supplements on muscle hemodynamics, and similar to our findings, reported no effect on muscle oxygen saturation [17].

Our study was also the first to examine the effects of constituent ingredients compared to the 'cocktail' supplement. L-arginine alpha-ketoglutarate is the major ingredient in Xpand Nitric Oxide Reactor, and it is converted to L-arginine in vivo. Furthermore, supplementation with $\mathrm{L}$-arginine alpha-ketoglutarate has been shown to increase plasma levels of $\mathrm{L}$ arginine [26], and L-arginine is a precursor for nitric oxide synthesis [27]. But similar to recent studies by Tang et al. (2011) and Willoughby et al. (2011), we found no effect of AAKG supplementation on hemodynamics. Tang et al. (2011) found no increase in lower-body muscle blood flow at rest or after resistance exercise following bolus arginine supplementation compared to placebo [28], and Willoughby et al. (2011) reported no change in upper-body muscle blood flow following 7 days of L-arginine alpha-ketoglutarate supplementation with resistance exercise [26]. It is still possible, however, that the L-arginine alphaketoglutarate supplementation in our study was not sufficient enough to increase $\mathrm{NO}$, thus potentially explaining the lack of change in hemodynamic characteristics in the muscle. Increased vasodilation in humans has been demonstrated with supraphysiological doses of arginine $(30 \mathrm{~g})$ that were administered intraveneously [29]. But we used doses consistent with Xpand Nitric Oxide Reactor for all supplement treatments (ranging from $\sim 4$ to $8 \mathrm{~g}$ ).

Caffeine, the other major ingredient in Xpand Nitric Oxide Reactor, also did not significantly affect soleus muscle re-oxygenation half-time after exercise. Flow mediated dilatation has been shown to be impaired up to 60 minutes after caffeine ingestion [7]. In our study, caffeine was ingested 45 minutes prior to exercise, and perhaps not enough time was permitted for impairment of endothelial function. However, our recorded caffeine supplementation half-times were consistently the longest amongst all subjects, and our results were trending towards significance.

\section{SUMMARY}

In summary, we examined the effects of preexercise supplementation with an L-arginine containing 'cocktail' supplement compared to two main ingredients, caffeine and L-arginine alphaketoglutarate, on calf muscle re-oxygenation during and after 3 sets of intense resistance exercise. Our study is the first to examine L-arginine alpha- 
ketoglutarate supplementation on hemodynamics in the soleus muscle of the leg, and our results are in agreement with previous findings. Even though arginine-containing 'cocktail' supplements may improve physical performances, there is growing evidence that these supplements have little to no effect on muscle hemodynamics. Thus, any ergogenic effects of these supplements are likely to not be related to improvements in blood flow nor re-oxygenation, instead creatine monohydrate or caffeine may have been responsible for previous reports of increased performance with arginine-containing 'cocktail' supplements. It seems possible that by combining ingredients for the purpose of optimizing physical performance may actually cause a wash-out of the intended physiological benefit of increased muscle blood flow because of interference between ingredients.

\section{ACKNOWLEDGEMENTS}

The authors would like to thank the study volunteers for their time and effort, and Kevin McCully, Ph.D., Robert Klug, M.D., Wayne Shelton, Dale Hubbard, John Kecman, Christina Orcino, Kevin Palmer, Troy Grube, and Matthew Overstreet for their assistance throughout the project. This project was supported in part by the Department of Health and Sport Sciences, Salisbury University and a University Student Academic Research Award (USARA).

\section{REFERENCES}

[1] Alvares TS, Meirelles CM, Bhambhani YN, Paschoalin VMF, Gomes PSC. L-arginine as a potential ergogenic aid in healthy subjects. Sports Med 2011; 41(3): 233-48. http://dx.doi.org/10.2165/11538590-000000000-00000

Maiorana A, O'Driscoll G, Taylor R, Green D. Exercise and the nitric oxide vasodilator system. Sports Med 2003; 33: 1013-35.

http://dx.doi.org/10.2165/00007256-200333140-00001

[3] Goud C, DiPiero A, Lockette W, Webb R, Charpie J. Cyclic GMP-independent mechanisms of nitric oxide induced vasodilation. Gen Pharmacol 1998; 32: 51-5. http://dx.doi.org/10.1016/S0306-3623(98)00059-7

Shibata M, Ichioka S, Kamiya A. Nitric oxide modulates oxygen consumption by arteriolar walls in rat skeletal muscle. Am J Physiol Heart Circ Physiol 2005; 289(6): 2673-9. http://dx.doi.org/10.1152/ajpheart.00420.2005

[5] Böger $\mathrm{RH}$. The pharmacodynamics of L-arginine. J Nutr 2007; 137(6): 1650-5

[6] Little J, Forbes S, Candow D, Cornish S, Chilibeck P. Creatine, arginine $\alpha$-ketoglutarate, amino acids, and mediumchain triglycerides and endurance and performance. Int $\mathrm{J}$ Sport Nutr Exerc Metab 2008; 18(5): 493-508.

[7] Tarnopolsky M. Caffeine and creatine use in sport. Ann Nutr Metab 2010; 57: 1-8. http://dx.doi.org/10.1159/000322696
Paddon-Jones D, Børsheim E, Wolfe RR. Potential ergogenic effects of arginine and creatine supplementation. J Nutr 2004; 134(10): 2888S-94S.

[9] Rahimi R. Creatine supplementation decreases oxidative DNA damage and lipid peroxidation induced by a single bout of resistance exercise. J Strength Cond Res 2011; 25(12): 3448-55.

http://dx.doi.org/10.1519/JSC.0b013e3182162f2b

[10] Schoch R, Willoughby D, Greenwood M. The regulation and expression of the creatine transporter: a brief review of creatine supplementation in humans and animals. J Int Soc Sports Nutr 2006; 3(1): 60-6. http://dx.doi.org/10.1186/1550-2783-3-1-60

[11] Volek JS, Kraemer WJ, Bush JA, Boetes M, Incledon T, Clark KL, et al. Creatine supplementation enhances muscular performance during high-intensity resistance exercise. J Am Diet Assoc 1997; 97(7): 765-70. http://dx.doi.org/10.1016/S0002-8223(97)00189-2

[12] Gotshalk LA, Volek JS, Staron RS, Deneger CR, Hagerman FC, Kraemer WJ. Creatine supplementation improves muscular performance in older men. Med Sci Sports Exerc 2002; 34(3): 537-43. http://dx.doi.org/10.1097/00005768-200203000-00023

[13] Campbell B, Roberts M, Kerksick C, Wilborn C, Marcello B Taylor L, et al. Pharmacokinetics, safety, and effects on exercise performance of $\mathrm{L}$-arginine $\alpha$-ketoglutarate in trained adult men. Nutrition 2006; 22(9): 872-81. http://dx.doi.org/10.1016/j.nut.2006.06.003

[14] McAllister R, Newcomer S, Laughlin M. Vascular nitric oxide: effects of exercise training in animals. Appl Physiology Nutr Metab 2008; 33(1): 173-8. http://dx.doi.org/10.1139/H07-146

[15] McConell GK. Effects of L-arginine supplementation on exercise metabolism. Curr Opin Clin Nutr Metab Care 2007; 10(1): 46-51.

http://dx.doi.org/10.1097/MCO.0b013e32801162fa

[16] Papamichael CM, Aznaouridis KA, Karatzis EN, Karatzi KN Stamatelopoulos KS, Vamvakou G, et al. Effect of coffee on endothelial function in healthy subjects: the role of caffeine. Clin Sci 2005; 109(1): 55-60. http://dx.doi.org/10.1042/CS20040358

[17] Bloomer RJ, Farney TM, Trepanowski JF, McCarthy CG, Canale RE, Schilling BK. J Strength Cond Res. Comparison of pre-workout nitric oxide stimulating dietary supplements on skeletal muscle oxygen saturation, blood nitrate/nitrite, lipid peroxidation, and upper body exercise performance in resistance trained men. J Int Soc Sports Nutr 2010; 7: 6-17. http://dx.doi.org/10.1186/1550-2783-7-16

[18] Baechle T, Earle R. Essentials of Strength Training and Conditioning 3rd ed. Champaign, Illinois: Human Kinetics 2008.

[19] Belardinelli R, Barstow T, Porszasz J, Wasserman K Changes in skeletal muscle oxygenation during incremental exercise measured with near infrared spectroscopy. Eur $J$ Appl Physiol Occup Physiol 1995; 70(6): 487-92. http://dx.doi.org/10.1007/BF00634377

[20] Ferrari M, Binzoni T, Quaresima V. Oxidative metabolism in muscle. Philos Trans R Soc Lond B Biol Sci 1997; 352(1354): 677-83

http://dx.doi.org/10.1098/rstb.1997.0049

[21] Mancini D, Wilson J, Bolinger L, Li H, Kendrick K, Chance B, et al. In vivo magnetic resonance spectroscopy measurement of deoxymyoglobin during exercise in patients with heart failure. Demonstration of abnormal muscle metabolism despite adequate oxygenation. Circulation 1994 90(1): 500-8.

http://dx.doi.org/10.1161/01.CIR.90.1.500 
[22] Rundell KW, Nioka S, Chance B. Hemoglobin/myoglobin desaturation during speed skating. Med Sci Sports Exer $1997 ; 29(2): 248-58$ http://dx.doi.org/10.1097/00005768-199702000-00014

[23] Wilson J, Mancini D, McCully K, Ferraro N, Lanoce V, Chance B. Noninvasive detection of skeletal muscle underperfusion with near-infrared spectroscopy in patients with heart failure. Circulation 1989; 80(6): 1668-74. http://dx.doi.org/10.1161/01.CIR.80.6.1668

[24] Prosser JM, Majlesi N, Chan GM, Olsen D, Hoffman RS, Nelson LS. Adverse effects associated with arginine alphaketoglutarate containing supplements. Hum Exp Toxicol 2009; 28(5): 259-62. http://dx.doi.org/10.1177/0960327109104498

[25] Bescós R, Sureda A, Tur JA, Pons A. The effect of nitricoxide-related supplements on human performance. Sports Med 2012; 42(2): 99-117. http://dx.doi.org/10.2165/11596860-000000000-00000

[26] Willoughby DS, Boucher T, Reid J, Skelton G, Clark M. Effects of 7 days of arginine-alpha-ketoglutarate supplementation on blood flow, plasma L-arginine, nitric oxide metabolites, and asymmetric dimethyl arginine after resistance exercise. Int J Sport Nutr Exerc Metab 2011; 21(4): 291-9.

[27] Palmer RM, Ashton DS, Moncada S. Vascular endothelial cells synthesize nitric oxide from L-arginine. Nature 1988; 333(6174): 664-6.

http://dx.doi.org/10.1038/333664a0

[28] Tang JE, Lysecki PJ, Manolakos JJ, MacDonald MJ, Tarnopolsky MA, Phillips SM. Bolus arginine supplementation affects neither muscle blood flow nor muscle protein synthesis in young men at rest or after resistance exercise. J Nutr 2011; 141(2): 195-200. http://dx.doi.org/10.3945/jn.110.130138

[29] Bode-Böger SM, Böger RH, Galland A, Tsikas D, Frölich JC. L-arginine-induced vasodilation in healthy humans: pharmacokinetic-pharmacodynamic relationship. $\mathrm{Br} \mathrm{J}$ Clin Pharmacol 1998; 46(5): 489-97.

http://dx.doi.org/10.1046/j.1365-2125.1998.00803.x

\section{DOl: http://dx.doi.org/10.6000/1929-5634.2012.01.02.5}

(c) 2012 Collins et al.; Licensee Lifescience Global.

This is an open access article licensed under the terms of the Creative Commons Attribution Non-Commercial License (http://creativecommons.org/licenses/by-nc/3.0/) which permits unrestricted, non-commercial use, distribution and reproduction in any medium, provided the work is properly cited. 